\title{
La calcolosi renale infetta e la calcolosi renale con infezione: terapia preventiva, in particolare post-chirurgica
}

\author{
A. Cupisti, V. Marchetti, C. D’Alessandro, G. Sbragia, G. Barsotti \\ Nefrologia Universitaria, Dipartimento di Medicina Interna, Università di Pisa
}

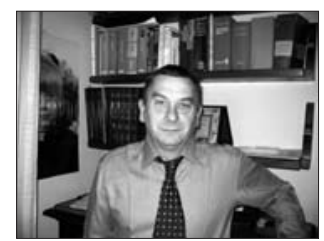

A. Cupisti

La calcolosi renale infetta, o di struvite, è una forma meno frequente rispetto a quella calcica o di acido urico, ma è molto più aggressiva e può essere responsabile di progressione verso l'insufficienza renale. Tuttavia qualsiasi tipo di litiasi, in particolare se recidivante e/o complicata da uropatia ostruttiva o da infezione, può costituire una potenziale causa di danno parenchimale renale irreversibile. Nello studio retrospettivo di Jungers (1), riguardante 1391 pazienti incidenti in dialisi tra il 1989 ed il 2000, la calcolosi renale rappresenta la malattia di base nel 3.2\% dei casi; in questo gruppo di pazienti, la calcolosi di struvite era presente nel $42 \%$ dei pazienti, la calcolosi calcica nel $26.7 \%$, quella urica nel $17.7 \%$, mentre il $13 \%$ dei pazienti era affetto da una forma ereditaria di nefrolitiasi.

E noto che la calcolosi renale è un fattore di rischio di infezione urinaria che, a sua volta, favorisce la formazione di calcoli. Quindi la sepsi urinaria e la calcolosi renale sono due patologie che possono auto-alimentarsi in un vero e proprio circolo vizioso di non facile trattamento. La presenza di infezione è stata riportata nel $32 \%$ delle calcolosi di ossalato di calcio (2), mentre Mariappan et al (3) hanno riportato come nel $25 \%$ dei casi di uropatia ostruttiva si riscontrasse la presenza di infezione del calcolo, e che un'infezione del tratto urinario a monte dell'ostruzione era presente fino al 66\% dei casi.

Quando a colonizzare le vie escretrici sono germi ureasi-produttori, è l'infezione stessa che causa modificazioni della composizione delle urine tali da portare alla formazione di cristalli e quindi di calcoli di triplo fosfato di ammonio e magnesio e carbonato apatite, sale anche chiamato "struvite". Il termine "struvite" fu coniato nel 1920 dal chimico Georg Ludwig Ulex in onore di Hein- rich Christoph Gottfried von Struve, un diplomatico con la grande passione per i minerali.

La struvite è una forma di calcolosi infetta che consegue allinfezione delle vie escretrici da parte di germi capaci di produrre un enzima non presente nell'uomo: l'ureasi. L'ureasi di derivazione batterica idrolizza l'urea presente nelle urine in ammoniaca ed anidride carbonica che, reagendo con l'acqua, danno origine alla formazione di ioni ammonio e bicarbonato, con conseguente elevazione del pH. Si determina così una condizione unica, non presente in fisiologia e caratterizzata da una elevata concentrazione di ioni ammonio in presenza di $\mathrm{pH}$ alcalino, che conduce alla nucleazione del triplo fosfato di ammonio e magnesio e carbonato apatite, sale mai presente nelle urine normali (4). Dei patogeni responsabili delle infezioni delle vie urinarie, quelli produttori di ureasi sono Providencia R, Morganella M, Proteus V e Klebsiella P in oltre il 95\% dei casi; seguiti da Yersinia E, Stafilococco A e Proteus $M$ nel 90\% dei casi e da Enterobacter spp (50\%), Pseudomonas A (33\%), mentre nel caso della Escherichia coli solo l'1\% dei ceppi produce l'ureasi.

Contrariamente a quanto sarebbe logico aspettarsi, il riscontro di sepsi urinaria nel paziente con calcolosi di struvite non è un reperto costante nella pratica clinica. Nello studio di Hakagashi et al (5), la positività dell'urinocoltura veniva riscontrata solo nel $20 \%$ delle calcolosi di struvite, con una positività dell'esame microbiologico del calcolo che arrivava fino al 50\% dei casi. Questa "strana" negatività dell'esame colturale, potrebbe essere giustificata da una bassa carica microbica o dalla presenza di un "biofilm". Il biofilm è costituito da aggregati batterici inglobati in una matrice di polisaccaridi, prodotta dai batteri stessi, in grado di aderire sia ai tessuti biologici sia a materiali sintetici, e che conferiscono ai germi la capacità di sopravvivere in ambienti ostili. Molte infezioni croniche risultano, infatti, difficilmente eradicabili 
dalla terapia antibiotica proprio per la presenza del biofilm. L'esistenza del biofilm può giustificare le colture falsamente negative oppure conte batteriche sottostimate (6). Di fronte ad una calcolosi di struvite con urinocoltura ripetutamente negativa deve anche essere ricercata la presenza di germi non evidenziabili con l'urinocoltura "per germi comuni”, ma comunque responsabili della formazione di struvite, quali i Micoplasmi e i Corinebatteri, ed in particolare l'Ureoplasma ureolitico (7).

I fattori di rischio per le infezioni delle vie urinarie sono l'età avanzata, la malnutrizione, il diabete mellito, una ridotta risposta del sistema immunitario, il fumo, l'obesità, la presenza di infezioni in altre sedi, le malformazioni delle vie urinarie, l'uropatia ostruttiva, la calcolosi, gli interventi urologici e la presenza di drenaggi o cateteri vescicali. Inoltre, il rischio di contrarre un'infezione urinaria è elevato in corso di ospedalizzazione; Bierklund in un lavoro del 2006 (8), evidenziava su 6033 pazienti ricoverati in ambiente urologico, una prevalenza di infezioni delle vie urinarie del $12 \%$, nella maggior parte dei casi sostenute da $E$. coli ma, in buona parte, sostenute anche da germi più difficili e spesso resistenti ai comuni antibiotici, come le Pseudomonas e gli Enterococchi.

Il legame tra metabolismo minerale e microbiologia si osserva anche nella recente scoperta dei nanobatteri, cioè di microrganismi da 10 a 100 volte più piccoli dei normali batteri che giocherebbero un ruolo nella formazione dei nidi di nucleazione di cristalli e, quindi, di calcoli. Esistono evidenze in letteratura che i nanobatteri, il cui ruolo rimane ad oggi comunque controverso, siano in grado di produrre carbonato-apatite diventando, quindi, essi stessi responsabili della formazioni di calcoli infetti, di recidiva di malattia e reazioni infiammatorie acute sistemiche (9).

La calcolosi di struvite, la calcolosi con infezione, la batteriuria pelvica e l'uropatia ostruttiva, sono fattori di rischio per complicanze settiche, anche molto gravi, in particolare in rapporto ai necessari interventi urologici. Prevenire lo sviluppo di una complicanza infettiva, cioè di una urosepsi è molto importante ma non sempre facile. Questa complicanza può manifestarsi anche in presenza di un'urinocoltura pre-operatoria negativa e nonostante una terapia antibiotica profilattica.

L'uso di chinolonici (ciprofloxacina $250 \mathrm{mg}$ x 2 al giorno) nella settimana precedente l'intervento di pielolitotomia percutanea in pazienti con calcolosi a stampo di dimensioni maggiori di $2 \mathrm{~cm}$ e dilatazione pielocaliceale, riduceva di tre volte il rischio di sviluppare un'infezione delle alte vie urinarie e della metà l'incidenza di positività della coltura del calcolo (10); inoltre nel gruppo trattato con chinolonico vi era un rischio 3 volte inferiore di sviluppare una grave uro- sepsi (10) durante il decorso post-intervento.

I dati della letteratura suggeriscono che nei pazienti che presentano una uropatia ostruttiva, un calcolo di grosse dimensioni, una coltura positiva o che siano affetti da diabete mellito, è indicata una terapia antibiotica profilattica con chinolonici o cefalosporine da 2 a 7 giorni prima dellintervento di litotrissia percutanea e/o rimozione endoscopica del calcolo (11).

Per quanto riguarda il trattamento della calcolosi renale tramite litotrissia extracorporea, è importante considerare che il trauma può favorire il passaggio di germi dalle urine al circolo sistemico, con rischio di sepsi, soprattutto laddove vi sia un'urinocoltura pre-trattamento positiva e/o un'uropatia ostruttiva. In questi casi è sicuramente indicata una terapia antibiotica come sopra specificato. In uno studio prospettico (3) è stata valutata la relazione tra la coltura del calcolo e le colture di campioni di urina raccolti con la minzione o prelevati direttamente dalla pelvi, e lo sviluppo di urosepsi. Dei 54 pazienti sottoposti a nefrolitotomia percutanea, solo 6 avevano una urinocoltura (vescicale) positiva contro gli 11 positivi per la coltura delle urine pelviche e i 19 positivi per la coltura del calcolo. I pazienti con infezione dell'urina pelvica o del calcolo sono risultati avere un rischio di sepsi 4 volte maggiore; mentre l'infezione dell'urina vescicale non è risultata predittiva. In particolare la positività della coltura del calcolo presentava la maggior predittività positiva ed i germi più frequentemente isolati erano E. coli, Proteus e Stafilococchi coagulasi negativi.

Un altro interessante studio (12) ha valutato la correlazione tra l'esito dell'urinocoltura (su urina vescicale) pre-operatoria e la coltura del calcolo nei pazienti sottoposti a nefrolitotomia percutanea. Di questi, circa il $50 \%$ presentavano negatività ed il $24 \%$ positività di entrambi i campioni. Il 25\% dei pazienti mostrava, invece, una coltura positiva del calcolo ma urinocoltura negativa. La sensibilità e specificità dell'urinocoltura come indicatore di positività del calcolo sono risultate rispettivamente del 30 e del $94 \%$, con una predittività positiva dell' $84 \%$ e negativa del $58 \%$. Inoltre in 13 dei 17 pazienti che hanno sviluppato urosepsi la scelta dell'antibiotico è stata modificata con successo sulla base dell'antibiogramma della coltura del calcolo.

Riassumendo, i dati della letteratura evidenziano l'importanza della prevenzione e del trattamento delle infezioni delle vie urinarie, e che la presenza di un calcolo infetto è la causa principale di complicanze settiche post-intervento urologico. Data la scarsa sensibilità e predittività dell'urinocoltura per g.c. sul campione vescicale, sarebbe auspicabile la regolare applicazione di una procedura che preveda la coltura del calcolo rimosso e delle urine pelviche (o a monte dell'ostruzione), con 
esecuzione dell'antibiogramma, elementi che potranno rappresentare un utile strumento di guida per le successive decisioni terapeutiche.

Il trattamento di scelta della calcolosi infetta rimane la completa rimozione del calcolo, essendo i frammenti residui fonte persistente d'infezione e nucleo di aggregazione di nuovi cristalli con recidiva di malattia. In ogni caso sarà indicato eseguire terapia antibiotica profilattica con chinolonici o cefalosporine 1-2 giorni prima o nei 7 giorni precedenti l'intervento urologico ed eseguire la coltura delle urine pelviche e del calcolo per un miglior inquadramento etiologico e per scelta terapeutica più mirata nella fase post-chirurgica.

\author{
Indirizzo degli Autori: \\ Adamasco Cupisti, MD \\ Nefrologia Universitaria \\ Dipartimento di Medicina Interna \\ Università di Pisa \\ Pisa, Italy \\ acupisti@int.med.unipi.it
}

\section{Bibliografia}

1. Jungers P, Joly D, Barbey F, Choukroun G, Daudon M. ESRD caused by nephrolithiasis: prevalence, mechanisms and prevention. Am J Kidney Dis 2004; 44(5): 799-805.

2. Hugusson J, Grenabo L, Hedelin H, Pettersson S, Seeberg S. Bacteriology of upper urinary tract stones. J Urol 1990; 143: 965-8.

3. Mariappan P, Smith G, Bariol SV, Moussa SA, Tolley DA. Stone and pelvic urine colture and sensitivity are better than bladder urine as predictors of urosepsis following percutaneous nephrolithotomy: a prospective clinical study. J Urol 2005; 173: 1610-4.

4. Bichler KH, Eipper E, Naber K, Braun V, Zimmermann $\mathrm{R}$, Lahme S. Urinary infection stones. Int J Antimicrob Agents 2002; 19: 488-98.

5. Akagashi K, Tanda H, Kato S et al. Characteristics of patients with staghorn calculi in our experience. Int J Urol 2004; 11: 276-81.

6. Aparna MS, Yadav S. Biofilms: microbes and disease. Braz J Infect dis 2008; 12: 526-30.

7. Grenabo L, Hedelin H, Petterson S. Urinary infection stones caused by Ureaplasma urealyticum: a review. Scand J
Infect Dis 1988; 53(Suppl): S46-9.

8. Johansen TE, Cek M, Naber KG, et al. Hospital acquired urinary tract infections in urology departments: pathogens, susceptibility and use of antibiotics. Data from the PEP and PEAP-studies. Int J Antimicrob Agents 2006; 28 (Suppl 1): S91-107.

9. Kajander EO, Ciftçioglu N. Nanobacteria: an alternative mechanism for pathogenic intra- and extracellular calcification and stone formation. Proc Natl Acad Sci USA 1998; 95: 8274-9.

10. Mariappan P, Smith G, Moussa SA, Tolley DA. One week of ciprofloxacin before percutaneous nephrolithotomy significantly reduces upper tract infection and urosepsis: a prospective controlled study. BJU Int 2006; 98: 1075-9.

11. Zanetti G, Paparella S, Trinchieri A, Prezioso D, Rocco F, Naber KG. Infections and Urolithiasis: current clinical evidence in prophylaxis and antibiotic therapy. Archivio Italiano di Urologia e Andrologia 2008; 80: 5-12.

12. Margel D, Ehrlich Y, Brown N, Lask D, Livne PM, Lifshitz DA. Clinical implication of routine stone culture in percutaneous nephrolithotomy: a prospective study. Urology 2006; 67: 26-9 